\title{
Relationships between the diabetes awareness and clinical indices/nutrient intakes in Korean adults: Based on the 2012-2013 Korea National Health and Nutrition Examination Survey Data
}

\author{
Seul Lee', Haeryun Park', Youngmi Lee', Onjeong Choi ${ }^{1}$, Jiwon Kim ${ }^{1}$, Heewon L Gray ${ }^{2}$ and Kyunghee Song ${ }^{15}$ \\ ${ }^{1}$ Department of Food and Nutrition, Myongji University, Yongin, Gyeonggi 17058, Korea \\ ${ }^{2}$ Department of Community and Family Health, University of South Florida 13201, Florida, USA.
}

BACKGROUND/OBJECTIVES: Diabetes is diagnosed after an average of 10-12 years of diabetic development. Strict glycemic control in diabetic patients promotes the normalization of blood glucose and reduces cardiovascular diseases (CVDs) and diabetic complications. Therefore, early diagnosis in non-aware individuals is very important.

SUBJECTS/METHODS: Clinical indices and nutrient intakes in Korean diabetic adults aged 19-64 years were examined according to the awareness of diabetes, using 2012 and 2013 Korea National Health and Nutrition Examination Survey (KNHANES) data. The aware group was defined as individuals who were aware of having diabetes from diagnosis by physician before the survey and the non-aware group as individuals who were not aware of having diabetes.

RESULTS: The average age was higher in the aware group compared to the non-aware group in both men ( $P=0.002)$ and women $(P=0.004)$. The prevalences of hypertension and dyslipidemia were not different between the two groups, but the diagnosis rate was significantly lower in the non-aware group. In the non-aware group, total and LDL-cholesterol were significantly higher $(P<0.001)$, the risk for total cholesterol over $240 \mathrm{mg} / \mathrm{dL}$ was 3.4 times $(95 \% \mathrm{Cl}$ : 1.58-7.52) higher $(P=0.002)$ and the risk for LDL-cholesterol over $160 \mathrm{mg} / \mathrm{dL}$ was 4.59 times (95\% Cl: $2.07-10.17)$ higher $(P<0.001)$. The calorie intake of the female non-aware group was significantly higher compared to the female aware group $(P=0.033)$.

CONCLUSION: The results suggested that the recommendation of screening test is necessary even for young adults. Studies on the methodology for early diagnosis of diabetes are also needed.

Nutrition Research and Practice 2019;13(3):240-246; https://doi.org/10.4162/nrp.2019.13.3.240; pISSN 1976-1457 elSSN 2005-6168

Keywords: Diabetes, aware, clinical indices, nutrient intake

\section{INTRODUCTION}

Diabetes is one of chronic diseases and the mortality rate caused by diabetes has increased every year worldwide [1]. Diabetes is a major risk factor for cardiocerebrovascular disease, which is a major cause of death in diabetic patients [2]. When $\mathrm{HbA} 1 \mathrm{c}$ (6.5\% and over) was included in the diagnostic criteria for diabetes, the prevalence of diabetes has increased in both men and women in Korea recently [3].

Only strict glycemic control can prevent complications of diabetes [4]. According to the guidelines for diabetes by the American Diabetes Association (ADA), controlling BMl, $\mathrm{HbA1c}$, blood pressure and cholesterol is recommended [5]. Based on the Korean diabetes management guidelines, $\mathrm{HbA} 1 \mathrm{c}<6.5 \%$, blood pressure $\leq 140 / 80 \mathrm{mmHg}$, LDL-cholesterol $\leq 100 \mathrm{mg} / \mathrm{dL}$, $\mathrm{TG} \leq 150 \mathrm{mg} / \mathrm{dL}$ and $\mathrm{HDL}$-cholesterol $\geq 40 \mathrm{mg} / \mathrm{dL}$ for men and $\geq 50 \mathrm{mg} / \mathrm{dL}$ for women are recommended [6].

Pharmacotherapy, dietary therapy and exercise therapy are commonly used to manage diabetes. Dietary therapy plays an important role in delaying the development of diabetic complications during the course of treatment and progression of diabetes. Proper dietary therapy improves metabolic abnormalities and blood lipid profiles [7,8]. Studies on nutrient intakes in Korean diabetic patients showed that the intake of carbohydrates was more than $60 \%$ and even higher in women than in men $[9,10]$. The increased carbohydrate intake causes hyperglycemia, which can develop oxidative stress in the body and finally cause tissue damage [11]. In addition, excessive insulin secretion can increase body fat to induce obesity [12].

Among diabetic complications, microvascular complications are increased along with an increased duration of diabetes, while macrovascular complications are affected by the risk factors for cardiovascular diseases (CVDs) [8]. In addition, the prevalence of CVDs is more than 2-4 times higher in diabetic patients compared to non-diabetic patients $[13,14]$. The awareness of diabetes in Korea tends to be higher in women than in men. In particular, the awareness rate was the lowest in men in their $30 \mathrm{~s}$ and $40 \mathrm{~s}$ [3].

\footnotetext{
${ }^{\S}$ Corresponding Author: Kyunghee Song, Tel. 82-31-330-6206, Fax. 82-31-330-6200, Email. khsong@mju.ac.kr

Received: February 18, 2019, Revised: March 18, 2019, Accepted: May 20, 2019

This is an Open Access article distributed under the terms of the Creative Commons Attribution Non-Commercial License (http://creativecommons.org/licenses/by-nc/3.0/ which permits unrestricted non-commercial use, distribution, and reproduction in any medium, provided the original work is properly cited.
} 
According to a study by the American Medical Care System, the onset of diabetes, on average, is up to 10-12 years before clinical diagnosis [15]. Complications such as diabetic retinopathy or diabetic neuropathy are commonly found at the time of diagnosis [16] and CVDs such as peripheral arterial disease and coronary heart disease are co-diagnosed in many cases [17]. Therefore, early diagnosis is very important to prevent complications and comorbidities of diabetes, yet associations among patients' awareness of diabetes, its clinical indicators, and nutrient intakes have not been well investigated in the Korean population, especially using nationally representative data.

The purpose of this study was to examine clinical indices and nutrient intakes based on diabetes awareness among adults aged 19-64 years, using nationally representative data from the 2012-2013 Korea National Health and Nutrition Examination Survey (KNHANES).

\section{SUBJECTS AND METHODS}

\section{Study design and participants}

A cross-sectional secondary analysis was conducted using the 2012-2013 KNHANES data. Participants who were 19-64 years old and diagnosed with diabetes at the time of the measurement were included. For diabetes indicators, fasting blood glucose and $\mathrm{HbA} 1 \mathrm{c}$ were used from the hematology section of the KNHANES. A total of 523 participants were divided into two groups: (1) the aware group (297; 148 men and 149 women), defined as individuals who were diagnosed by medical doctors; and (2) the non-aware group (226; 103 men and 123 women), defined as individuals who were not clinically diagnosed at the time of the measurement but had 8-h fasting plasma glucose over $126 \mathrm{mg} / \mathrm{dL}$ or $\mathrm{HbA} 1 \mathrm{c}$ over $6.5 \%$. The Institutional Review Board (IRB) of the Korea Center for Disease Control and Prevention approved the KNHANES (IRB. 201201EXP-01-2C and 2013-07CON-03-4C).

\section{General characteristics}

Information on gender, age, income level, education level, exercise, drinking and smoking patterns was obtained from the KNHANES data. The exercise patterns were classified into two levels: moderate/walking was classified as more than 10 minutes per session and more than 20 minutes of daily exercise for at least 3 days a week and vigorous exercise was classified as more than 10 minutes per session and more than 30 minutes of daily exercise for at least 5 days a week. Drinking pattern was classified into a binary variable: either equal to greater vs. fewer than once a month during the past one year. Height, weight and waist circumference data were extracted from the anthropometric measurement section of the KNHANES.

\section{Prevalence and diagnosis of hypertension and dyslipidemia}

The prevalence of hypertension was defined as systolic pressure $\geq 140 \mathrm{mmHg}$ or diastolic pressure $\geq 90 \mathrm{mmHg}$. The prevalence of dyslipidemia was defined as total cholesterol $\geq$ $240 \mathrm{mg} / \mathrm{dL}$ or triglyceride $\geq 200 \mathrm{mg} / \mathrm{dL}$ or HDL-cholesterol < $40 \mathrm{mg} / \mathrm{dL}$ or LDL-cholesterol $\geq 160 \mathrm{mg} / \mathrm{dL}$. The diagnosis rate for each of hypertension and dyslipidemia was defined as individuals who were diagnosed by a medical doctor. LDL- cholesterol was calculated with the Friedewald equation for those with serum triglyceride level $\leq 400 \mathrm{mg} / \mathrm{dL}$ [18].

\section{Clinical indices}

The following data were obtained from the health examination survey: systolic pressure, diastolic pressure, total-cholesterol, HDL-cholesterol, triglyceride, hemoglobin, hematocrit, blood urea nitrogen (BUN) and blood creatinine.

\section{Nutrient intakes}

The intake of nutrients was analyzed through the type and amount of food consumed the day before the survey using the $24 \mathrm{~h}$ recall method. The ratio of intakes of protein, fat and carbohydrates was calculated using the presented nutrient values. For the analysis of the intake ratio by nutrients, the Dietary Reference Intakes for Koreans (2010) was used for different age groups.

\section{Statistical analysis}

Statistical analysis was performed after adjusting for the KNHANES sample weights, strata and clusters. Clinical indices and nutrient intakes were analyzed after controlling for age. Group comparisons were performed based on the aware vs. non-aware groups. Independent samples t-tests were performed for continuous data and $\mathrm{x}^{2}$-tests were performed for nominal data. General linear models were used to examine differences in biochemical indices, energy and nutrient intakes by group, after controlling for age. To analyze the proper ratio of protein fat and carbohydrates intake by group, logistic regressions were performed. Odds ratio (OR) and 95\% confidence interval (Cl) are presented. All data in the study were analyzed using SPSS 19.0 (SPSS Inc., Chicago, USA) and the statistical significances were tested at $P<0.05$.

\section{RESULTS}

\section{General characteristics}

The general characteristics of the participants by gender and diabetes awareness are shown in Table 1. The average ages of men and women were significantly higher at 53.2 and 53.6 years in the aware group compared to 48.8 and 49.3 years in the non-aware group $(P=0.002$ and $P=0.004)$, respectively. There were significant differences in the distribution of age categories between the aware and the non-aware groups. A large percentage of men and women in the non-aware group was in their $50 \mathrm{~s}(41.6 \%)$ and $60 \mathrm{~s}$ or older $(23.5 \%)$, while $43.1 \%$ of the aware group was in their $60 \mathrm{~s}$ or older $(P=0.001)$. The average BMI in men was significantly higher at $25.8 \mathrm{~kg} / \mathrm{m}^{2}$ in the non-aware group compared to $24.7 \mathrm{~kg} / \mathrm{m}^{2}$ in the aware group $(P=0.031)$. The average $\mathrm{BMl}$ in women was not significantly different between two groups, but the distribution of BMI was significantly different $(P=0.026)$. The obesity rate was $50.3 \%$ in the female aware group and $64.2 \%$ in the female non-aware group.

Prevalence and diagnosis of hypertension and dyslipidemia

The comorbidity status of hypertension and dyslipidemia is shown in Table 2. The prevalence of hypertension in women 
Table 1. General characteristics of subjects by sex and diabetes awareness.

\begin{tabular}{|c|c|c|c|c|c|c|c|c|c|}
\hline & \multicolumn{3}{|c|}{ Male $(n=251)$} & \multicolumn{3}{|c|}{ Female $(n=272)$} & \multicolumn{3}{|c|}{ Total $(n=523)$} \\
\hline & $\begin{array}{c}A G^{1)} \\
(n=148)\end{array}$ & $\begin{array}{c}\mathrm{NAG}^{2)} \\
(\mathrm{n}=103)\end{array}$ & $P$-value & $\begin{array}{c}A G \\
(n=149)\end{array}$ & $\begin{array}{c}\text { NAG } \\
(n=123)\end{array}$ & $P$-value & $\begin{array}{c}\text { AG } \\
(n=297)\end{array}$ & $\begin{array}{c}\text { NAG } \\
(n=226)\end{array}$ & $P$-value \\
\hline Age (yrs) & $53.2 \pm 1.0^{3)}$ & $48.8 \pm 1.0$ & $0.002^{* *}$ & $53.6 \pm 0.9$ & $49.3 \pm 1.3$ & $0.004^{* *}$ & $53.4 \pm 0.7$ & $49.0 \pm 0.8$ & $0.001^{* * *}$ \\
\hline $19-29$ & $2(1.4)^{4)}$ & $3(2.9)$ & $0.028^{*}$ & $2(1.3)$ & $4(3.3)$ & $0.009^{* *}$ & $4(1.3)$ & $7(3.1)$ & $0.001^{* * *}$ \\
\hline $30-39$ & $6(4.1)$ & $11(10.7)$ & & $4(2.7)$ & $11(8.9)$ & & $10(3.4)$ & $22(9.7)$ & \\
\hline $40-49$ & $21(14.2)$ & $23(22.3)$ & & $21(14.1)$ & $27(22.0)$ & & $42(14.1)$ & $50(22.1)$ & \\
\hline $50-59$ & $54(36.5)$ & $41(39.8)$ & & $59(39.6)$ & $53(43.1)$ & & $113(38.0)$ & $94(41.6)$ & \\
\hline$\geq 60$ & 65 (43.9) & $25(24.3)$ & & $63(42.3)$ & $28(22.8)$ & & $128(43.1)$ & $53(23.5)$ & \\
\hline Height (cm) & $169.3 \pm 0.7$ & $171.3 \pm 0.7$ & $0.024^{*}$ & $155.1 \pm 0.5$ & $158.0 \pm 0.7$ & $0.001^{* *}$ & $163.0 \pm 0.7$ & $165.8 \pm 0.6$ & $0.003^{* *}$ \\
\hline Weight $(\mathrm{cm})$ & $71.0 \pm 1.2$ & $76.0 \pm 1.5$ & $0.009^{* *}$ & $63.5 \pm 1.8$ & $69.3 \pm 2.0$ & $0.034^{*}$ & $67.7 \pm 1.0$ & $73.2 \pm 1.2$ & $0.001^{* * *}$ \\
\hline Waist circumference $(\mathrm{cm})$ & $86.9 \pm 0.8$ & $88.8 \pm 1.2$ & 0.159 & $86.8 \pm 1.5$ & $88.3 \pm 1.4$ & 0.468 & $86.8 \pm 0.8$ & $88.6 \pm 0.9$ & 0.148 \\
\hline BMI $\left(\mathrm{kg} / \mathrm{m}^{2}\right)^{5)}$ & $24.7 \pm 0.3$ & $25.8 \pm 0.4$ & $0.031^{*}$ & $26.4 \pm 0.7$ & $27.6 \pm 0.7$ & 0.217 & $25.5 \pm 0.4$ & $26.6 \pm 0.4$ & $0.047^{*}$ \\
\hline Underweight & $1(0.7)$ & $1(1.0)$ & 0.076 & $3(2.0)$ & $1(0.8)$ & $0.026^{*}$ & $4(1.35)$ & $2(0.9)$ & $0.006^{* *}$ \\
\hline Normal & $41(27.7)$ & $21(20.4)$ & & $40(26.8)$ & $16(13.0)$ & & $81(27.3)$ & $37(16.4)$ & \\
\hline Overweight & $46(31.1)$ & 19 (18.4) & & $31(20.8)$ & $27(22.0)$ & & 77 (25.9) & $46(20.4)$ & \\
\hline Obesity & $60(40.5)$ & $62(60.2)$ & & $75(50.3)$ & $79(64.2)$ & & $135(45.5)$ & $141(62.4)$ & \\
\hline \multicolumn{10}{|l|}{ House income } \\
\hline Low & $19(12.8)$ & $11(10.7)$ & 0.463 & $34(22.8)$ & $18(14.6)$ & 0.491 & $53(17.8)$ & $29(12.8)$ & 0.317 \\
\hline Middle-low & $34(23.0)$ & $24(23.3)$ & & $56(37.6)$ & $36(29.3)$ & & $90(30.3)$ & $60(26.5)$ & \\
\hline Middle-high & $44(29.7)$ & $30(29.1)$ & & $34(22.8)$ & $41(33.3)$ & & $78(26.3)$ & 71 (31.4) & \\
\hline High & $51(34.5)$ & 38 (36.9) & & $25(16.8)$ & $28(22.8)$ & & $76(25.6)$ & $66(29.2)$ & \\
\hline \multicolumn{10}{|l|}{ Education } \\
\hline$\leq$ Elementary school & $36(24.3)$ & $13(12.6)$ & $0.016^{*}$ & 58 (38.9) & $33(26.8)$ & 0.267 & 94 (31.6) & $46(20.4)$ & $0.019^{*}$ \\
\hline Middle school & $26(17.6)$ & $12(11.7)$ & & $25(16.8)$ & $30(24.4)$ & & $51(17.2)$ & $42(18.6)$ & \\
\hline High school & $41(27.7)$ & 39 (37.9) & & $51(34.2)$ & $38(30.9)$ & & $92(31.0)$ & $77(34.1)$ & \\
\hline$\geq$ College & $45(30.4)$ & 39 (37.9) & & $15(10.1)$ & $22(17.9)$ & & $60(20.2)$ & $61(27.0)$ & \\
\hline \multicolumn{10}{|l|}{ Exercise } \\
\hline Yes & $72(48.6)$ & 39 (37.9) & 0.126 & $65(43.6)$ & $53(43.1)$ & 0.763 & $160(53.9)$ & $134(59.3)$ & 0.293 \\
\hline No & $76(51.4)$ & $64(62.1)$ & & $84(56.4)$ & $70(57.0)$ & & $137(46.1)$ & $92(40.7)$ & \\
\hline \multicolumn{10}{|l|}{ Smoking } \\
\hline Current & 54 (36.5) & $48(46.6)$ & 0.085 & $6(4.0)$ & $6(4.9)$ & 0.589 & $157(52.9)$ & $131(58.0)$ & 0.052 \\
\hline Ex-smoker & $75(50.7)$ & $40(38.8)$ & & $5(3.4)$ & $1(0.8)$ & & $80(26.9)$ & $41(18.1)$ & \\
\hline None & $19(12.8)$ & 15 (14.6) & & $138(92.6)$ & $116(94.3)$ & & $60(20.2)$ & $54(23.9)$ & \\
\hline \multicolumn{10}{|l|}{ Drinking } \\
\hline Yes & $44(29.7)$ & $24(23.3)$ & 0.375 & $38(25.5)$ & $44(35.8)$ & 0.187 & $155(52.2)$ & $103(45.6)$ & 0.103 \\
\hline No & $104(70.3)$ & $79(76.7)$ & & $111(74.5)$ & $79(64.2)$ & & $142(47.8)$ & $123(54.4)$ & \\
\hline Fasting blood glucose (mg) & $139.6 \pm 3.8$ & $136.7 \pm 3.6$ & 0.526 & $151.9 \pm 5.6$ & $138.4 \pm 5.2$ & 0.094 & $145.3 \pm 3.4$ & $137.3 \pm 3.1$ & 0.081 \\
\hline $\mathrm{HbA1C}(\%)$ & $7.4 \pm 0.2$ & $6.9 \pm 0.1$ & $0.017^{*}$ & $7.9 \pm 0.2$ & $7.4 \pm 0.2$ & $0.038^{*}$ & $7.6 \pm 0.1$ & $7.1 \pm 0.1$ & $0.002^{*}$ \\
\hline
\end{tabular}

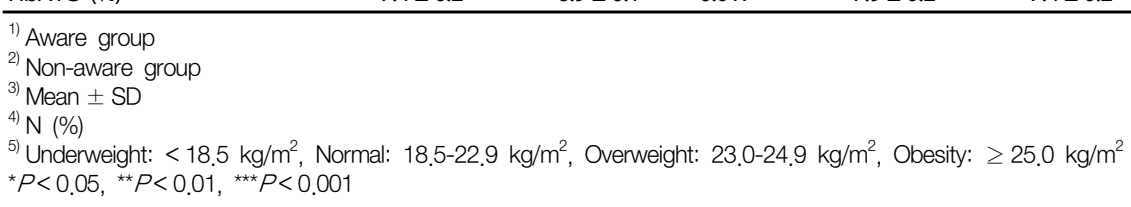

Table 2. Prevalence and diagnosis of hypertension and dyslipidemia of subjects by sex and diabetes awareness.

\begin{tabular}{|c|c|c|c|c|c|c|c|c|c|}
\hline & \multicolumn{3}{|c|}{ Male $(n=251)$} & \multicolumn{3}{|c|}{ Female $(n=272)$} & \multicolumn{3}{|c|}{ Total $(n=523)$} \\
\hline & $\begin{array}{c}A G^{1)} \\
(n=148)\end{array}$ & $\begin{array}{c}N^{\left.N A G^{2}\right)} \\
(n=103)\end{array}$ & $P$-value & $\begin{array}{c}\text { AG } \\
(n=149)\end{array}$ & $\begin{array}{c}\text { NAG } \\
(n=123)\end{array}$ & $P$-value & $\begin{array}{c}\text { AG } \\
(n=297)\end{array}$ & $\begin{array}{c}\text { NAG } \\
(n=226)\end{array}$ & $P$-value \\
\hline Prevalence of hypertension ${ }^{3)}$ & $72(48.6)^{4)}$ & $50(48.5)$ & 0.425 & $82(55.0)$ & $44(35.8)$ & $0.013^{*}$ & 154 (51.9) & 94 (41.6) & 0.417 \\
\hline Diagnosed of hypertension & $66(44.6)$ & $26(25.2)$ & $0.024^{*}$ & $79(53.0)$ & $37(30.1)$ & $0.008^{* *}$ & $145(48.8)$ & $63(27.9)$ & $0.001^{* *}$ \\
\hline Prevalence of dyslipidemia ${ }^{5)}$ & $93(62.8)$ & $70(68.0)$ & 0.210 & $98(65.8)$ & $80(65.0)$ & 0.755 & $191(64.3)$ & $150(66.4)$ & 0.218 \\
\hline Diagnosed of dyslipidemia & $46(31.1)$ & $12(11.7)$ & $0.001^{* * *}$ & $70(47.0)$ & $21(17.1)$ & $0.001^{* * *}$ & $116(39.1)$ & $33(14.6)$ & $0.001^{* * *}$ \\
\hline
\end{tabular}

\footnotetext{
1) Aware group

2) Non-aware group

3) Prevalence of hypertension: systolic pressure $\geq 140 \mathrm{mmHg}$ or diastolic pressure $\geq 90 \mathrm{mmHg}$

4) $\mathrm{N}(\%)$

5) Prevalence of dyslipidemia: Total Cholesterol $\geq 240 \mathrm{mg} / \mathrm{dL}$ or HDL-Cholesterol $<40 \mathrm{mg} / \mathrm{dL}$ or LDL-Cholesterol $\geq 60 \mathrm{mg} / \mathrm{dL}$ or Triglyceride $\geq 200 \mathrm{mg} / \mathrm{dL}$

${ }^{\star} P<0.05,{ }^{* \star} P<0.01,{ }^{* \star *} P<0.001$
} 
Table 3. The biochemical indices of subjects by sex and diabetes awareness.

\begin{tabular}{|c|c|c|c|c|c|c|c|c|c|}
\hline & \multicolumn{3}{|c|}{ Male $(n=251)$} & \multicolumn{3}{|c|}{ Female $(n=272)$} & \multicolumn{3}{|c|}{ Total $(n=523)$} \\
\hline & $\begin{array}{c}A G^{1)} \\
(n=148)\end{array}$ & $\begin{array}{c}\mathrm{NAG}^{2)} \\
(\mathrm{n}=103)\end{array}$ & $P$-value & $\begin{array}{c}\text { AG } \\
(n=149)\end{array}$ & $\begin{array}{c}\text { NAG } \\
(n=123)\end{array}$ & $P$-value & $\begin{array}{c}\text { AG } \\
(n=297)\end{array}$ & $\begin{array}{c}\text { NAG } \\
(n=226)\end{array}$ & $P$-value \\
\hline Systolic pressure & $124.3 \pm 1.5^{3)}$ & $126.2 \pm 1.8$ & 0.424 & $121.4 \pm 1.6$ & $122.3 \pm 1.6$ & 0.698 & $123.0 \pm 1.2$ & $124.6 \pm 1.3$ & 0.368 \\
\hline Diastolic pressure & $78.7 \pm 1.0$ & $83.3 \pm 1.4$ & $0.010^{*}$ & $74.9 \pm 1.0$ & $78.9 \pm 0.9$ & $0.004^{* *}$ & $77.0 \pm 0.8$ & $81.5 \pm 0.9$ & $0.001^{* * *}$ \\
\hline Total cholesterol (mg/dL) & $177.4 \pm 3.7$ & $201.1 \pm 3.8$ & $0.001^{* * *}$ & $182.0 \pm 3.8$ & $214.2 \pm 4.4$ & $0.001^{* * *}$ & $179.4 \pm 2.6$ & $206.6 \pm 2.9$ & $0.001^{* * *}$ \\
\hline HDL-cholesterol (mg/dL) & $44.2 \pm 1.0$ & $42.7 \pm 1.1$ & 0.333 & $46.3 \pm 1.1$ & $46.9 \pm 1.1$ & 0.686 & $45.1 \pm 0.7$ & $44.5 \pm 0.8$ & 0.547 \\
\hline LDL-cholesterol (mg/dL) & $102.5 \pm 3.2$ & $118.6 \pm 3.3$ & $0.001^{* * *}$ & $106.1 \pm 3.5$ & $136.3 \pm 4.2$ & $0.001^{* * *}$ & $104.0 \pm 2.3$ & $126.0 \pm 2.7$ & $0.001^{* * *}$ \\
\hline Triglyceride (mg/dL) & $153.8 \pm 7.6$ & $199.3 \pm 10.1$ & $0.001^{* * *}$ & $148.0 \pm 7.1$ & $155.0 \pm 7.3$ & 0.481 & $151.3 \pm 5.3$ & $180.8 \pm 7.0$ & $0.001^{* * *}$ \\
\hline Hemoglobin (g/dL) & $14.9 \pm 0.1$ & $15.5 \pm 0.1$ & $0.002^{* *}$ & $13.0 \pm 0.2$ & $13.6 \pm 0.1$ & $0.019^{*}$ & $14.0 \pm 0.1$ & $14.7 \pm 0.1$ & $0.002^{* *}$ \\
\hline Hematocrit (\%) & $43.6 \pm 0.3$ & $45.2 \pm 0.4$ & $0.002^{* *}$ & $39.1 \pm 0.5$ & $40.8 \pm 0.3$ & $0.006^{* *}$ & $41.6 \pm 0.4$ & $43.4 \pm 0.3$ & $0.001^{* * *}$ \\
\hline BUN (mg/dL) & $16.3 \pm 0.4$ & $15.4 \pm 0.4$ & 0.157 & $15.5 \pm 1.3$ & $13.9 \pm 0.4$ & 0.292 & $15.9 \pm 0.6$ & $14.8 \pm 0.3$ & 0.125 \\
\hline Creatinine (mg/dL) & $1.2 \pm 0.0$ & $1.0 \pm 0.0$ & $0.001^{* * *}$ & $0.9 \pm 0.1$ & $0.7 \pm 0.0$ & 0.200 & $1.0 \pm 0.1$ & $0.9 \pm 0.0$ & $0.008^{* *}$ \\
\hline
\end{tabular}

Adjusted for age

1) Aware group

${ }^{2)}$ Non-aware group

3) Mean $+\mathrm{SD}$

${ }^{\star} P<0.05,{ }^{* *} P<0.01,{ }^{* *} P<0.001$

Table 4. Comparison of nutrient intake of subjects by sex and diabetes awareness.

\begin{tabular}{|c|c|c|c|c|c|c|c|c|c|}
\hline & \multicolumn{3}{|c|}{ Male $(n=251)$} & \multicolumn{3}{|c|}{ Female $(n=272)$} & \multicolumn{3}{|c|}{ Total $(n=523)$} \\
\hline & $\begin{array}{c}A G^{1)} \\
(n=148)\end{array}$ & $\begin{array}{c}\left.\mathrm{NAG}^{2}\right) \\
(\mathrm{n}=103)\end{array}$ & $P$-value & $\begin{array}{c}A G \\
(n=149)\end{array}$ & $\begin{array}{c}\text { NAG } \\
(n=123)\end{array}$ & $P$-value & $\begin{array}{c}\text { AG } \\
(n=297)\end{array}$ & $\begin{array}{c}\text { NAG } \\
(n=226)\end{array}$ & $P$-value \\
\hline Energy (kcal) & $2251 \pm 77^{3)}$ & $2452 \pm 132$ & 0.172 & $1665 \pm 74$ & $1891 \pm 69$ & $0.033^{*}$ & $1990 \pm 60$ & $2218 \pm 84$ & $0.025^{*}$ \\
\hline Protein (g) & $78.5 \pm 3.3$ & $90.6 \pm 6.3$ & 0.096 & $61.3 \pm 3.7$ & $68.8 \pm 4.3$ & 0.196 & $70.9 \pm 2.4$ & $81.5 \pm 4.2$ & $0.038^{*}$ \\
\hline Fat $(g)$ & $43.2 \pm 2.5$ & $44.7 \pm 3.8$ & 0.739 & $33.1 \pm 2.7$ & $37.4 \pm 2.4$ & 0.267 & $38.7 \pm 1.9$ & $41.6 \pm 2.5$ & 0.342 \\
\hline Carbohydrate (g) & $341.4 \pm 11.5$ & $362.7 \pm 18.6$ & 0.284 & $280.7 \pm 13.0$ & $319.2 \pm 13.3$ & $0.048^{*}$ & $314.3 \pm 9.6$ & $344.6 \pm 12.4$ & $0.044^{*}$ \\
\hline Fiber (g) & $9.3 \pm 0.5$ & $8.5 \pm 0.6$ & 0.391 & $7.7 \pm 0.6$ & $7.9 \pm 0.5$ & 0.717 & $8.5 \pm 0.4$ & $8.3 \pm 0.4$ & 0.669 \\
\hline Vitamin A (mg) & $919.2 \pm 103.5$ & $744.4 \pm 61.1$ & 0.160 & $754.1 \pm 116.3$ & $954.0 \pm 102.3$ & 0.167 & $844.5 \pm 77.1$ & $833.0 \pm 57.3$ & 0.902 \\
\hline Vitamin $B_{1}(\mathrm{mg})$ & $1.8 \pm 0.1$ & $2.3 \pm 0.2$ & 0.054 & $1.5 \pm 0.1$ & $1.7 \pm 0.1$ & 0.130 & $1.7 \pm 0.1$ & $2.0 \pm 0.1$ & $0.020^{*}$ \\
\hline Vitamin $B_{2}(\mathrm{mg})$ & $1.4 \pm 0.1$ & $1.5 \pm 0.1$ & 0.441 & $1.1 \pm 0.1$ & $1.3 \pm 0.1$ & $0.042^{*}$ & $1.3 \pm 0.0$ & $1.4 \pm 0.1$ & 0.065 \\
\hline Niacin (mg) & $18.7 \pm 0.9$ & $19.9 \pm 1.2$ & 0.446 & $14.8 \pm 1.0$ & $15.8 \pm 0.8$ & 0.456 & $17.0 \pm 0.7$ & $18.2 \pm 0.8$ & 0.261 \\
\hline Vitamin C (mg) & $112.7 \pm 9.1$ & $127.8 \pm 15.9$ & 0.400 & $104.4 \pm 10.3$ & $126.5 \pm 13.0$ & 0.170 & $109.0 \pm 7.0$ & $127.3 \pm 10.9$ & 0.150 \\
\hline Calcium (mg) & $566.9 \pm 26.7$ & $533.5 \pm 33.7$ & 0.427 & $442.0 \pm 26.9$ & $499.9 \pm 38.3$ & 0.203 & $510.9 \pm 20.5$ & $519.9 \pm 25.6$ & 0.777 \\
\hline Phosphorus (mg) & $1,321.0 \pm 46.5$ & $1,336.1 \pm 65.8$ & 0.851 & $1,035.7 \pm 53.0$ & $1,071.0 \pm 46.5$ & 0.632 & $1,193.7 \pm 36.8$ & $1,226.0 \pm 44.8$ & 0.590 \\
\hline Sodium (mg) & $4,940.3 \pm 288.0$ & $5,132.2 \pm 317.2$ & 0.654 & $3,962.5 \pm 389.8$ & $4,576.0 \pm 350.2$ & 0.234 & $4,506.5 \pm 236.1$ & $4,899.0 \pm 235.5$ & 0.232 \\
\hline Potassium (mg) & $3,481.0 \pm 151.4$ & $3,456.4 \pm 171.6$ & 0.912 & $2,798.8 \pm 166.6$ & $3,140.6 \pm 230.5$ & 0.220 & $3,177.2 \pm 115.4$ & $3,325.1 \pm 139.6$ & 0.409 \\
\hline Iron (mg) & $25.9 \pm 7.1$ & $20.0 \pm 2.3$ & 0.448 & $14.5 \pm 0.8$ & $17.3 \pm 1.1$ & 0.050 & $20.8 \pm 4.0$ & $18.9 \pm 1.5$ & 0.654 \\
\hline \multicolumn{10}{|l|}{ Distribution } \\
\hline$\%$ Protein & $14.0 \pm 0.3$ & $14.7 \pm 0.6$ & 0.296 & $14.9 \pm 0.5$ & $14.4 \pm 0.6$ & 0.516 & $14.4 \pm 0.3$ & $14.6 \pm 0.4$ & 0.761 \\
\hline$\%$ Fat & $16.4 \pm 0.8$ & $16.0 \pm 0.9$ & 0.709 & $17.0 \pm 0.8$ & $17.6 \pm 0.9$ & 0.628 & $16.7 \pm 0.6$ & $16.7 \pm 0.7$ & 0.977 \\
\hline$\%$ Carbohydrate & $63.3 \pm 1.5$ & $62.2 \pm 1.8$ & 0.626 & $68.4 \pm 1.2$ & $67.7 \pm 1.2$ & 0.667 & $65.6 \pm 1.0$ & $64.5 \pm 1.2$ & 0.460 \\
\hline
\end{tabular}

Adjusted for age

1) Aware group

2) Non-aware group

3) Mean $\pm \mathrm{SD}$

* $P<0.05$

was significantly higher in the aware group (55.0\%) compared to $35.8 \%$ in the non-aware group $(P=0.013)$ and also more women in the aware group were clinically diagnosed with hypertension (53.0\%) compared to the non-aware group $(30.1 \%$; $P=0.008$ ). For men, there was no significant difference in prevalence but a significantly higher percentage of men in the aware group (44.6\%) had been clinically diagnosed with hypertension compared to the non-aware group (25.2\%; $P=0.024)$. The prevalence of dyslipidemia was not significantly different between the two groups. However, there were significant differences in the rate of clinical diagnosis of dyslipidemia in both men and women: in men, it was significantly higher in the aware group (31.1\%) compared to the non-aware group (11.7\%; $P=0.001)$; and in women, it was $47.0 \%$ in the aware group and $17.1 \%$ in the non-aware group $(P<0.001)$.

\section{Clinical indices}

The clinical indices of the subjects are shown in Table 3. Total 
Table 5. Percentage of nutrient intake of Korean Reference Nutrient Intake (RNI) by sex and diabetes awareness.

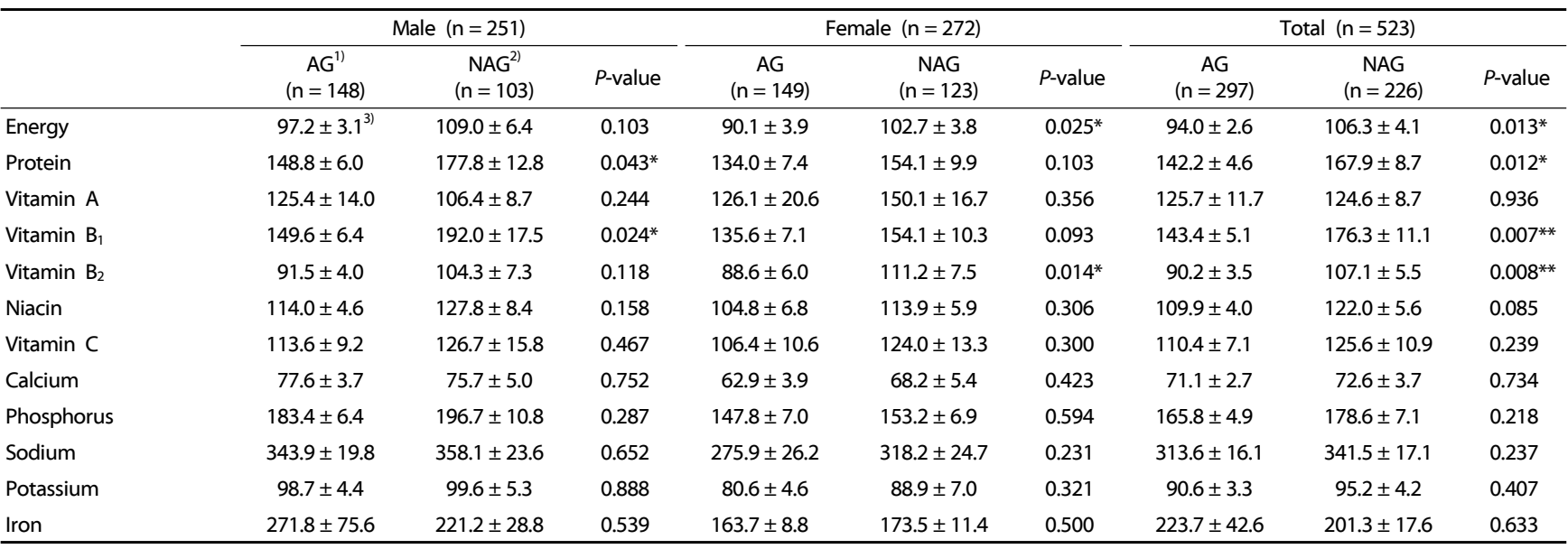

1) Aware group

2) Non-aware group

${ }^{3)}$ Mean $\pm \mathrm{SD}$

${ }^{*} P<0.05,{ }^{* *} P<0.01$

Table 6. Odds ratio $(\mathrm{OR})$ and $95 \%$ confidence interval $(\mathrm{Cl})$ of biochemical indices and nutrients intake distribution by diabetes awareness.

\begin{tabular}{|c|c|c|c|c|c|c|c|}
\hline & & $\begin{array}{c}\text { Crude OR } \\
(95 \% \mathrm{Cl})\end{array}$ & $P$-value & $\begin{array}{c}\text { Adjusted } \mathrm{OR}^{1)} \\
(95 \% \mathrm{Cl})\end{array}$ & $P$-value & $\begin{array}{c}\text { Adjusted } \mathrm{OR}^{2)} \\
(95 \% \mathrm{Cl})\end{array}$ & $P$-value \\
\hline \multirow[t]{2}{*}{ Waist circumference $M \geq 90 \mathrm{~cm}, \mathrm{~F} \geq 85 \mathrm{~cm}$} & Aware & 1 & & 1 & & 1 & \\
\hline & Non-aware & $1.93(1.25-2.98)$ & $0.003^{* *}$ & $2.25(1.30-3.90)$ & $0.004^{* *}$ & $2.64(1.18-5.88)$ & $0.018^{*}$ \\
\hline \multirow[t]{2}{*}{ Systolic pressure $\geq 140 \mathrm{mmHg}$} & Aware & 1 & & 1 & & 1 & \\
\hline & Non-aware & $0.89(0.48-1.64)$ & 0.710 & $1.26(0.59-2.68)$ & 0.548 & $1.25(0.60-2.60)$ & 0.545 \\
\hline \multirow[t]{2}{*}{ Diastolic pressure $\geq 90 \mathrm{mmHg}$} & Aware & 1 & & 1 & & 1 & \\
\hline & Non-aware & $2.10(1.16-3.8)$ & $0.015^{*}$ & $1.61(0.76-3.41)$ & 0.212 & $1.61(0.78-3.36)$ & 0.200 \\
\hline \multirow[t]{2}{*}{ Total cholesterol $\geq 240 \mathrm{mg} / \mathrm{dL}$} & Aware & 1 & & 1 & & 1 & \\
\hline & Non-aware & $4.08(2.04-8.14)$ & $0.001^{* * *}$ & $3.68(1.74-7.82)$ & $0.001^{* * *}$ & $3.44(1.58-7.52)$ & $0.002^{* *}$ \\
\hline \multirow[t]{2}{*}{$\mathrm{HDL}$-cholesterol $<40 \mathrm{mg} / \mathrm{dL}$} & Aware & 1 & & 1 & & 1 & \\
\hline & Non-aware & $1.46(0.95-2.26)$ & 0.088 & $1.67(0.96-2.92)$ & 0.071 & $1.62(0.93-2.81)$ & 0.088 \\
\hline \multirow[t]{2}{*}{ LDL-cholesterol $\geq 160 \mathrm{mg} / \mathrm{dL}$} & Aware & 1 & & 1 & & 1 & \\
\hline & Non-aware & $5.37(2.68-10.74)$ & $0.001^{* * *}$ & $4.46(2.05-9.07)$ & $0.001^{* * *}$ & $4.59(2.07-10.17)$ & $0.001^{* * *}$ \\
\hline \multirow[t]{2}{*}{ Triglyceride $\geq 200 \mathrm{mg} / \mathrm{dL}$} & Aware & 1 & & 1 & & 1 & \\
\hline & Non-aware & $1.98(1.23-3.17)$ & $0.005^{* *}$ & $2.14(1.17-3.09)$ & $0.013^{*}$ & $1.80(0.94-3.42)$ & 0.074 \\
\hline \multirow[t]{2}{*}{ Protein intake $>20 \%$} & Aware & 1 & & 1 & & 1 & \\
\hline & Non-aware & $0.89(0.37-2.12)$ & 0.791 & $0.73(0.26-20.4)$ & 0.540 & $0.77(0.24-2.82)$ & 0.666 \\
\hline \multirow[t]{2}{*}{ Fat intake $>25 \%$} & Aware & 1 & & 1 & & 1 & \\
\hline & Non-aware & $1.55(0.87-2.78)$ & 0.137 & $1.51(0.73-2.13)$ & 0.261 & $1.42(0.68-0.99)$ & 0.348 \\
\hline \multirow[t]{2}{*}{ Carbohydrate intake $>60 \%$} & Aware & 1 & & 1 & & 1 & \\
\hline & Non-aware & $0.76(0.47-1.22)$ & 0.250 & $0.79(0.43-1.46)$ & 0.448 & $0.94(0.48-1.85)$ & 0.860 \\
\hline
\end{tabular}

1) Adjusted for sex, age, income, education and diabetes duration

${ }^{2)}$ Adjusted for sex, age, income, education, diabetes duration, smoking, drinking, physical activity and BMI

cholesterol and LDL-cholesterol were significantly higher in the non-aware group compared to the aware group in both men and women $(P<0.001)$. In men, triglyceride was significantly higher at $199.3 \mathrm{mg} / \mathrm{dL}$ in the non-aware group compared to $153.8 \mathrm{mg} / \mathrm{dL}$ in the aware group $(P<0.001)$. Hemoglobin was $14.9 \mathrm{~g} / \mathrm{dL}$ in the aware group and $15.5 \mathrm{~g} / \mathrm{dL}$ in the non-aware group $(P=0.002)$. In women, hemoglobin was significantly lower in the aware group $(P=0.019)$. Hematocrit was significantly lower in the aware group compared to the non-aware group in both men $(P=0.002)$ and women $(P=0.006)$.

\section{Nutrient intakes}

Daily calorie and nutrient intakes are shown in Table 4. There was no significant difference between the aware and the non-aware groups among men. In women, calorie intake was $1,665 \mathrm{kcal}$ in the aware group and 1,891 kcal in the non-aware group $(P=0.033)$. Carbohydrate intake was significantly higher in the non-aware group (319.2 g) compared to the aware group (280.7 g; $P=0.048$ ). Vitamin $B_{2}$ intake was significantly higher in the non-aware group (1.3 $\mathrm{mg}$ ) compared to the aware group (1.1 $\mathrm{mg} ; P=0.042$ ) In total, calorie intake was $1,990 \mathrm{kcal}$ in the 
aware group and 2,218 kcal in the non-aware group $(P=0.025)$. Protein intake was significantly higher in the non-aware group $(81.5 \mathrm{~g})$ compared to the aware group (70.9 g; $P=0.038$ ). Carbohydrate intake was significantly higher in the non-aware group $(344.6 \mathrm{~g})$ compared to the aware group (314.3 g; $P=0.044$ ).

The results of nutrient intake of Korean Reference Nutrient Intake (RNI) of the subjects are shown in Table 5. The ratio of calorie intake to RNI was significantly higher at $106.3 \%$ in the non-aware group compared to $94.0 \%$ in the aware group ( $P$ $=0.013$ ), and both men and women in the non-aware group had an intake higher than Korean RNI. In men, protein intake was significantly higher at $177.8 \%$ in the non-aware group than $148.8 \%$ in the aware group ( $P=0.043)$. In men, vitamin $B_{1}$ intake was significantly higher in the non-aware group compared to the aware group $(P=0.024)$. In women, vitamin $B_{2}$ intake was significantly higher in the non-aware group compared to the aware group $(P=0.014)$.

The degree of risk exerted by diabetes awareness on clinical indices and nutrient intakes is shown in Table 6 . The crude risk for abnormal values was significantly higher for waist circumference, diastolic blood pressure, total cholesterol, LDLcholesterol and triglycerides in the non-aware group. After controlling for gender, age, income, education level, and the duration of diabetes, the risk for abnormal values was significantly higher for waist circumference, total cholesterol, LDL-cholesterol and triglycerides in the non-aware group. After additional correction for smoking, drinking, exercise and BMI, the risk for waist circumference over $90 \mathrm{~cm}$ in men and over $85 \mathrm{~cm}$ in women was 2.64 times (95\% Cl: 1.18-5.88) higher in the non-aware group $(P=0.018)$; and the risk for total cholesterol over $240 \mathrm{mg} / \mathrm{dL}$ was 3.44 times (95\% Cl: 1.58-7.52) higher in the non-aware group $(P=0.002)$. The risk for LDL-cholesterol over $160 \mathrm{mg} / \mathrm{dL}$ was 4.59 times (95\% Cl: 2.07-10.17) higher in the non-aware group $(P<0.001)$. Among nutrient intakes, abnormal intake ratios of protein, fat and carbohydrates were not significantly different.

\section{DISCUSSION}

This is the first study analyzing the health-related habits, clinical indices and nutrient intakes based on the diabetes awareness in the Korean adult population using nationally representative data (2012-2013 KNHANES) [3]. Early diagnosis and treatment of diabetes significantly reduces the risks for CVDs [19]. Therefore, providing men in their 30s with diabetes education would benefit the population health in Korea by reducing the prevalence and enhancing early treatment of diabetes in young men.

BMl was significantly higher in the non-aware group compared to the aware group and the ratio of BMl over $25 \mathrm{~kg} / \mathrm{m}^{2}$ was $45.5 \%$ and $62.4 \%$ in the aware and non-aware groups, respectively. Obesity is a major preceding factor of diabetes and a factor for preventing and delaying diabetes in individuals with the possibility of progressing diabetes. Weight control for obesity is needed in both the aware and non-aware groups.

Hypertension is a common comorbidity in diabetic patients and a major risk factor for CVDs [20,21]. Strict blood pressure control can reduce the mortality and delay complications such as diabetic retinopathy and reduced vision [22]. The study results indicated that the non-aware group was unaware of not only diabetes but also accompanying diseases such as hypertension and dyslipidemia. The diagnosis rate for hypertension and dyslipidemia was lower than the prevalence rate, demonstrating uncontrolled blood pressure and blood lipid profiles in the aware group. Screening and strict management of blood pressure, LDL-cholesterol, triglycerides and HDL-cholesterol are recommended for diabetic individuals. The results of this study were consistent with those in the studies on diabetic patients by Kwon et al. [23] and on diabetes awareness by Kim et al. [5]. Cardiocerebrovascular diseases rank as the $2^{\text {nd }}$ and $3^{\text {rd }}$ causes of death in Korea and individuals with diabetes have higher risks for CVDs [24]. Because the prevalence of CVD is 2-4 times higher in diabetic patients than in non-diabetic patients, early diagnosis in the non-aware group can promote the regulation of risk factors for CVDs through glycemic control and blood lipid control $[25,26]$.

Park et al. [27] showed a significant relationship between carbohydrate intake and waist circumference after controlling for total energy intake and BMI. The ADA recommends that the total amount rather than the type of carbohydrates is important in the intake of carbohydrates. Thus, education on carbohydrate control is highly recommended.

Lim et al. [28] reported that 'Healthy Korean' meal patterns, including whole grains, beans, nuts, vegetables, mushrooms and fruits significantly reduced serum cholesterol and triglycerides values, while 'bread, meat and alcohol' meal patterns significantly increased total cholesterol. Thus, planned lifestyle modifications including exercise and the control of energy intake are necessary for diabetic patients.

Limitations of the study include that the definition of the non-aware group was based on either fasting blood glucose or HbA1c data from the KNHANES. Because it relied on one measurement for each individual, it is possible that some participants might have been miss-categorized as diabetic.

Although the currently recommended target for diabetes screening is individuals over 40 years or over 30 years in case of having diabetic risk factors, this study suggested that diabetes screening before 40 years of age through regular health check-up would be beneficial. In the aware group, continuous and systematic management and education are necessary to prevent diabetic complications. Careful self-monitoring for glycemic control is also recommended for diabetic patients. To increase overall diabetes awareness, not only individual-level education but also social marketing or other community-level education strategies will be beneficial. Further studies determining effective strategies to increase diabetes awareness and screening as well as rigorous diabetes management programs are needed.

\section{CONFLICT OF INTEREST}

The authors declare no potential conflicts of interests.

\section{ORCID}

Seul Lee: https://orcid.org/0000-0003-4145-9865 
Haeryun Park: https://orcid.org/0000-0003-2627-6518

Youngmi Lee: https://orcid.org/0000-0001-9549-0716

Onjeong Choi: https://orcid.org/0000-0001-5093-8476

Jiwon Kim: https://orcid.org/0000-0002-3307-1441

Heewon L Gray: https://orcid.org/0000-0002-5257-5012

Kyunghee Song: https://orcid.org/0000-0001-9549-0716

\section{REFERENCES}

1. World Health Organization. The-top-10-causes-of-death[internet]. Geneva: World Health Organization; 2018 [cited 2019 May 27]. Available from: https://www.who.int/news-room/fact-sheets/detail/ the-top-10-causes-of-death.

2. Bloomgarden ZT. Diabetes and cardiovascular disease. Diabetes Care 2011;34:e24-30.

3. Korean Diabetes Association. Diabetes Fact Sheet in Korea [Internet]. Seoul: Korean Diabetes Association; 2018 [cited 2019 May 27]. Available from: http://www.diabetes.or.kr/pro/news/admin.php? category $=A \&$ code $=a d m i n \&$ number $=1428 \&$ mode $=$ view .

4. Gregg EW, Cadwell BL, Cheng YJ, Cowie CC, Williams DE, Geiss L, Engelgau MM, Vinicor F. Trends in the prevalence and ratio of diagnosed to undiagnosed diabetes according to obesity levels in the U.S. Diabetes Care 2004;27:2806-12.

5. Kim SM, Lee JS, Lee J, Na JK, Han JH, Yoon DK, Baik SH, Choi DS, Choi KM. Prevalence of diabetes and impaired fasting glucose in Korea: Korean National Health and Nutrition Survey 2001. Diabetes Care 2006;29:226-31.

6. Korean Diabetes Association. Treatment Guideline for Diabetes, 5th ed [Internet]. Seoul: Korean Diabetes Association; 2015 [cited 2019 May 27]. Available from: http://www.diabetes.or.kr/pro/publish/ guide.php?code=guide\&mode=list\&year_v=2015.

7. Franz MJ, Bantle JP, Beebe CA, Brunzell JD, Chiasson JL, Garg A, Holzmeister LA, Hoogwerf B, Mayer-Davis E, Mooradian AD, Purnell JQ, Wheeler M. Evidence-based nutrition principles and recommendations for the treatment and prevention of diabetes and related complications. Diabetes Care 2002;25:148-98.

8. Lim JH, Lee YS, Chang HC, Moon MK, Song Y. Association between dietary patterns and blood lipid profiles in Korean adults with type 2 diabetes. J Korean Med Sci 2011;26:1201-8.

9. Park YM, Sohn CM, Jang HC. Correlation of carbohydrate intake with obesity in type 2 diabetes mellitus patients. J Korean Diet Assoc 2006;12:254-63.

10. Kim EK, Lee JS, Hong HO, Yu CH. Association between glycemic index, glycemic load, dietary carbohydrates and diabetes from Korean National Health and Nutrition Examination Survey 2005. Korean J Nutr 2009;42:622-30.

11. Ceriello A, Testa R. Antioxidant anti-inflammatory treatment in type 2 diabetes. Diabetes Care 2009;32 Suppl 2:S232-6.

12. Weiss R, Dufour S, Taksali SE, Tamborlane WV, Petersen KF, Bonadonna RC, Boselli L, Barbetta G, Allen K, Rife F, Savoye M, Dziura J, Sherwin R, Shulman Gl, Caprio S. Prediabetes in obese youth: a syndrome of impaired glucose tolerance, severe insulin resistance, and altered myocellular and abdominal fat partitioning. Lancet 2003;362:951-7.

13. Girach A, Manner D, Porta M. Diabetic microvascular complications: can patients at risk be identified? A review. Int J Clin Pract 2006;60:1471-83.

14. Harris Ml, Klein R, Welborn TA, Knuiman MW. Onset of NIDDM occurs at least 4-7 yr before clinical diagnosis. Diabetes Care 1992;15:815-9.

15. UK Prospective Diabetes Study Group. UK Prospective Diabetes Study 6. Complications in newly diagnosed type 2 diabetic patients and their association with different clinical and biochemical risk factors. Diabetes Res 1990;13:1-11.

16. Siitonen $O$, Uusitupa $M$, Pyörälä $K$, Voutilainen $E$, Länsimies $E$. Peripheral arterial disease and its relationship to cardiovascular risk factors and coronary heart disease in newly diagnosed non-insulindependent diabetics. Acta Med Scand 1986;220:205-12.

17. Friedewald WT, Levy RI, Fredrickson DS. Estimation of the concentration of low-density lipoprotein cholesterol in plasma, without use of the preparative ultracentrifuge. Clin Chem 1972;18: 499-502.

18. Harris MI. Undiagnosed NIDDM: clinical and public health issues. Diabetes Care 1993;16:642-52.

19. Harris Ml, Eastman RC. Early detection of undiagnosed diabetes mellitus: a US perspective. Diabetes Metab Res Rev 2000;16:230-6.

20. Wingard DL, Barrett-Connor E. Heart disease and diabetes. In: Harris MI, Cowie CC, Stern MS, editors. Diabetes in America. Washington, D.C.: National Institutes of Health; 1995. p.429-48.

21. UK Prospective Diabetes Study Group. Tight blood pressure control and risk of macrovascular and microvascular complications in type 2 diabetes: UKPDS 38. BMJ 1998;317:703-13.

22. Arauz-Pacheco C, Parrott MA, Raskin P. The treatment of hypertension in adult patients with diabetes. Diabetes Care 2002;25:134-47.

23. Kwon JY, Chung HY. Study on the correlation between the nutrient intakes and clinical indices of type 2 diabetes patients. Korean J Food Nutr 2013;26:909-18.

24. Committee for Guidelines for Management of Dyslipidemia. 2015 Korean guidelines for management of dyslipidemia. J Lipid Atheroscler 2015;4:61-92.

25. Jee $S H$, Jang $Y$, Oh DJ, Oh BH, Lee $S H$, Park SW, Seung KB, Mok $Y$, Jung KJ, Kimm H, Yun YD, Baek SJ, Lee DC, Choi SH, Kim MJ, Sung J, Cho B, Kim ES, Yu BY, Lee TY, Kim JS, Lee YJ, Oh JK, Kim SH, Park JK, Koh SB, Park SB, Lee SY, Yoo Cl, Kim MC, Kim HK, Park JS, Kim HC, Lee GJ, Woodward M. A coronary heart disease prediction model: the Korean Heart Study. BMJ Open 2014;4: e005025.

26. Collins R, Armitage J, Parish S, Sleigh P, Peto R; Heart Protection Study Collaborative Group. MRC/BHF Heart Protection Study of cholesterol-lowering with simvastatin in 5963 people with diabetes: a randomised placebo-controlled trial. Lancet 2003;361:2005-16.

27. Park S, Park MS, Ko JA. The association between carbohydrate intake and waist circumference. Korean J Obes 2008;17:175-81. 